\title{
REVISIÓN DE ESQUEMAS DE CLASIFICACIÓN DE TEJIDOS CANCERÍGENOS EN IMÁGENES DE MAMOGRAFÍ́A
}

\author{
REVISION OF CLASSIFICATION SCHEMES \\ IN TISSUE CARCINOGENIC MAMMOGRAPHY IMAGES
}

\author{
YELEISY OCHOA', WILMER RIVAS', EDUARDO TUSA ${ }^{3}$, BERTHA MAZÓN ${ }^{4}$ \\ 1 Universidad Técnica de Machala.ymochoa_est@utmachala.edu.ec \\ 2 Universidad Técnica de Machala.wrivas@utmachala.edu.ec \\ 3 Universidad Técnica de Machala. etusa@utmachala.edu.ec \\ 4 Universidad Técnica de Machala. bmazon@utmachala.edu.ec
}

RESUMEN

La presente revisión de esquemas de clasificación analiza los algoritmos y métodos más utilizados dentro de la detección automática y clasificación supervisada de anomalías en imágenes de mamografías. Para ello, se propone el análisis de 36 artículos cientíicos en base a su metodología de clasificación, considerando además que estas publicaciones se encuentran indexadas en revistas reconocidas en los últimos cinco años. El estudio se ha dividido en cuatro etapas. La primera etapa es el preprocesamiento, que incluye las bases de datos utilizadas para entrenamiento y pruebas. Además, describimos los métodos de filtrados como la ecualización de histogramas (escala de grises) y el filtro Gabor, entre otros métodos que han brindado resultados eficientes. La etapa de los algoritmos de extracción de características presenta coincidencias en la utilización de la transformada discreta de coseno y la transformada discreta de ondícula entre otros algoritmos que se han utilizado para esta tarea. Para los algoritmos de clasificación, se resalta la utilización de las máquinas de vectores de soporte. En adición a la revisión de expresiones matemáticas desarrolladas en esta investigación, una de las contribuciones de este trabajo reside en proporcionar una lista de clasificadores híbridos, que resultan de la combinación de los clasificadores convencionales. Finalmente, se describen las métricas de evaluación como la curva receptora de características operativas y la precisión, que son indicadores cuantitativos del desempeño de los algoritmos revisados en este trabajo investigativo.

PALABRAS CLAVE: Algoritmo de clasificación, cáncer de mama, extracción de características, mamografía, reconocimiento de patrones.
The present revision of classification schemes analyzes the algorithms and methods most used within the automatic detection and supervised classification of abnormalities in mammography images. To do this, we propose the analysis of 36 scientific papers based on their classification methodology, considering also that these publications are indexed in recognized journals in the last five years. The study has been divided into four stages. The first stage is preprocessing, which includes databases used for training and testing. In addition, we describe the filtering methods such as histogram equalization (grayscale) and the Gabor filter, among other methods that have yielded efficient results. The stage of feature extraction algorithms presents coincidences in the use of the discrete cosine transform and the discrete wavelet transform, among other algorithms that have been used for this task. For the classification algorithms, we highlight the use of the support vector machine. In addition to the review of mathematical expressions developed in this research, one of the contributions of this paper is to provide a list of hybrid classifiers, which result from the combination of conventional classifiers. Finally, we describe the evaluation metrics such as the receiver operating characteristic curve and the precision, which are quantitative indicators of the performance of the algorithms reviewed in this research.

KEYWORDS: Algorithm classification, breast cancer, feature extraction, mammography, pattern recognition. 


\section{INTRODUCCIÓN}

En el año 2017, el cáncer de mama tuvo una presencia alarmante a nivel nacional e internacional, llegando a ocupar el segundo lugar entre los tipos de cáncer más ordinarios en las mujeres. Esto demuestra que por cada 10 diagnósticos de cáncer realizados, un cáncer positivo de mama fue detectado (Thomaz, Carneiro, \& Patrocinio, 2017). El incremento de los decesos por esta enfermedad, ha llamado la atención de las autoridades de salud en el Ecuador. Las cifras sobre el cáncer de mama de acuerdo a la base de datos de la Sociedad de Lucha Contra el Cáncer (SOLCA) de la ciudad de Guayaquil en el año 2012, presentó una tasa de mortalidad de 11 muertes por cada 100.000 habitantes, con una incidencia del $38 \%$ en las mujeres cuyas edades se encuentran entre 50 y 64 años (Torre et al., 2015). Frente a este escenario, existe la necesidad de mejorar los esquemas de diagnóstico.

La mamografía es el método de detección temprana más utilizado cuando no existen síntomas de por medio, contribuyendo al aumento de la expectativa de supervivencia del paciente. Con los últimos avances de la inteligencia artificial, los algoritmos de aprendizaje automático han alcanzado una gran acogida en el diagnóstico asistido por computador CAD, a través de tareas como: segmentación, detección de bordes, redimensión y ecualización de imagen; permitiendo que los algoritmos de reconocimiento puedan categorizar automáticamente los diagnósticos, ya sean estos: malignos, benignos y normales (Pérez, Benalcazar, Conci, \& Fiallos, 2016).

El objetivo de este trabajo es la revisión de esquemas de clasificación de tejidos cancerígenos en imágenes de mamografías mediante la descripción de diferentes etapas como el preprocesamiento, señalando las técnicas de filtrado que se aplican. En esta misma línea, se presenta las bases de datos de mamografías más utilizadas para la clasificación de diagnósticos. Además, se describe los algoritmos de extracción de características y de clasificación, identificando las métricas aplicadas para la evaluación de sus rendimientos. Con ello, se pretende sintetizar los métodos seleccionados, valorando su desempeño en el proceso de identificación de tejidos cancerígenos en las mamografías.

\section{PREPROCESAMIENTO DE LA IMAGEN}

En esta etapa, se busca mejorar la calidad de la imagen para asegurar un adecuado procesamiento de los datos durante la fase de clasificación (Pérez et al., 2016). A continuación, se describen las bases de datos más utilizadas, así como también; los filtros que se aplican para reducir la distorsión en las imágenes de mamografías.

\section{BASES DE DATOS}

La base de datos de la Sociedad de Análisis de Imágenes de Mamografías (MIAS, por sus siglas en inglés), fue elaborada por una asociación de investigadores que brindan aportes desde Gran Bretaña e Irlanda del Norte. La base de datos está comprendida por 322 imágenes digitalizadas que tiene como tamaño de $2.3 \mathrm{~GB}$, con una dimensión de 1024 X 1024 pixeles. Con una disminución de 200 micrones de pixeles, se pueden encontrar en un Archivo Piloto de Preprocesamiento de Imágenes Europeas (PEIPA, con sus siglas en ingles) en la Universidad de Essex (Malar, Kandaswamy, Chakravarthy, \& Giri Dharan, 2012).

La Base de Datos Digital para la Detección de Mamografía (DDSM, por sus siglas en inglés), se ha construido con la finalidad de brindar un apoyo a los investigadores conscientes de la necesidad de la detección de anomalías en imágenes de mamografías. Los involucrados en este proyecto son el Hospital General de Massachusetts, los Laboratorios Nacionales de Sandia y el Departamento de Ciencias de la Computación e Ingeniería de la Universidad del Sur de Florida. Cuenta con 2.500 diagnósticos donde muestran ambos senos debidamente ordenados con los datos del paciente: edad al momento del estudio, clasificación de la densidad mamaria ACR, calificación de su utilidad para las anormalidades (Khan, Mendes, Zhang, \& Chalup, 2017).

De acuerdo a los 36 artículos académicos que han sido revisados, 20 de ellos han presentado coincidencia con la base de datos MIAS, siendo esta la más utilizada. La base de datos DDSM presenta 11 coincidencias quedando en segundo lugar. A continuación, se presenta una tabla que muestra el resultado de las coincidencias sobre la revisión de los artículos.

TABLA 1. BASES DE DATOS MÁS UTILIZADAS PARA EL ESTUDIO DE CÁNCER DE MAMA

\begin{tabular}{lll}
\hline AUTORES & $\begin{array}{l}\text { NOMBRE DE LA } \\
\text { BASE DE DATOS }\end{array}$ & $\begin{array}{l}\text { NÚMERO DE } \\
\text { IMÁGENES } \\
\text { UTILIZADAS }\end{array}$ \\
\hline $\begin{array}{lll}\text { Edward J. Kendall* y cols., Mellisa Pratiwi } \\
\text { y cols., Arden Sagiterry Setiawan y cols., }\end{array}$ & $\begin{array}{l}\text { Mammographic } \\
\text { Image Analysis }\end{array}$ & 2017 \\
$\begin{array}{l}\text { Blagojce Jankulovski y cols., Maria G. } \\
\text { Perez y cols., Muhammad Talha, Pelin }\end{array}$ & $\begin{array}{l}\text { Society (MIAS) } \\
\text { (Unido, 2008) }\end{array}$ \\
$\begin{array}{l}\text { Gorgel y cols., Luqman Mahmood Mina } \\
\text { y cols., J. Subash Chandra Bose y cols., }\end{array}$ & \\
$\begin{array}{l}\text { Sharanya Padmanabhan y cols., loan Buciu } \\
\text { y cols., Mohamed Meselhy Eltoukhy y cols., }\end{array}$ & \\
Jae Young Choi y cols., Weiying Xie y cols., & & \\
\end{tabular}


Daniel Levy y cols., Saeid Asgari Taghanaki y cols., Salabat Khan y cols., Edward J.

Kendall ${ }^{*}$ y cols., Baljit Singh Khehra y

cols., loan Buciu y cols., Zhang Lifeng

y cols., Gabriele Magna y cols., Danays

Bárbara Costa Alonso y cols., Weiying Xie.,

Maryam Mahsal Khan y cols.,

El Sistema de Generación de Informes y Datos de Imágenes de Mama (BI-RADS, por sus siglas en inglés) fue elaborado por el Colegio Americano de Radiólogos como un aporte a la sociedad para la estandarización de los informes de mamografías. Mediante una estrategia de categorias de valores 0-5, se muestran los niveles de cáncer de mama clasificados según los niveles al que pertenece el diagnóstico establecido, tal como se aprecia en la Tabla 2.

\section{TABLA 2. CATEGORIZACIÓN Y DESCRIPTOR DE BI-RADS}

\begin{tabular}{|c|c|}
\hline $\begin{array}{l}\text { CATEGORÍA } \\
\text { DE BI-RADS }\end{array}$ & DESCRIPTOR DE BI-RADS \\
\hline 0 & Estudio incompleto \\
\hline 1 & Descubrimiento negativo \\
\hline 2 & Descubrimiento benignos \\
\hline 3 & $\begin{array}{l}\text { Descubrimiento probablemente benigno, seguir evaluación a } \\
\text { los } 6 \text { meses. }\end{array}$ \\
\hline 4 & $\begin{array}{l}\text { Descubrimiento sospechoso, es recomendable aplicar una } \\
\text { biopsia. }\end{array}$ \\
\hline 5 & Descubrimiento que sugiere firmemente la presencia de cáncer \\
\hline
\end{tabular}

Nota: Elaborado por el Colegio Americano de Radiólogos, (Radiología).

FILTROS DEL PREPROCESAMIENTO DE LA IMAGEN

El objetivo del preprocesamiento es mejorar la calidad de la imagen, para eliminar elementos que no forman parte de la información. Para cumplir este propósito, se realiza un filtrado sobre la imagen para prepararla para la fase de extracción de características.

ECUALIZACIÓN DEL HISTOGRAMA (ESCALA DE GRISES)

Es un método conocido por su estudio estadístico que aporta en la transformación de una imagen. Su propósito busca mejorar el contraste de una imagen tomando en cuenta todos los valores de intensidad. Para la adquisición de una distribución uniforme del histograma de la imagen, se aumenta el brillo y contraste de objetos muy brillantes u oscuros. Todo este proceso se lo realiza con la finalidad de obtener una imagen con un balance perfecto de los pixeles blancos y negros. Es de conocimiento general que los niveles de gris son agrupados entre 0 que es negro y 255 que es blanco (Pérez et al., 2016) . Dentro del grupo de artículos que se ha investigado, se aprecia que la ecualización de histogramas es un método bastante utilizado. A consecuencia de esto, algunos autores combinan esta ecualización con métodos externos para llegar al óptimo resultado.

La segmentación a través de un valor umbral (Método de Otsu), ayuda a fragmentar los pixeles de una imagen en escala de grises. Existen dos clases de valor de intensidad de umbral: umbral global, umbral local o adaptativo. El umbral global se denomina también como umbral fijo ( $\mathrm{T})$ pues genera una imagen con niveles de gris del pixel, cuyos valores están entre $(0,1)$ siendo una imagen binaria (Nariño, 2016). El umbral local o adaptativo se utiliza cuando la distribución es binomial a causa de la iluminación que se presenta. Según los autores en (Nariño, 2016), se debe fragmentar la imagen en subregiones para obtener un umbral óptimo para cada una de las partes implicadas. La asignación del umbral local no considera el tamaño o la forma del algoritmo, además se le asigna un umbral para cada subregión. Otra manera de estimar el valor umbral se explica al considerar el histograma bimodal y se itera a lo largo del histograma para calcular así la varianza de pesos dentro de cada clase o modo de Otsu. Cuando la varianza alcanza el valor mínimo entre dos clases, se obtiene el valor de umbral óptimo (Nariño, 2016).

TÉCNICA DE LIVE-WIRE

Esta técnica utilizada por (Díaz Sotolongo, Cárcel Carrasco, Martín Alfonso, \& Pérez Pérez, 2014), hace un seguimiento de bordes que permite la segmentación de la imagen con una mínima interacción. La ventaja es que tiene un operador de reconocimiento continuo al algoritmo de delineación automático. Este autor ha utilizado la técnica para obtener el contorno y centro de la lesión, que son empleados como primer paso para la detección de elementos de referencia de la imagen.

Esta técnica está compuesta por una función de costo local que agrega un costo menor a la característica de la imagen (bordes y expansión formando un límite entre los objetos de interés). El siguiente paso es la interpolación con la función spline para la representación de la curva continua que demuestra la aproximación del contorno del objeto. La curva extraída nos sirve para calcular los puntos de referencia (Pérez, Valdés, Guevara, Rodríguez, \& Molina, 2007).

FILTRO GAUSSIANO DOGS

En la investigación de (Madrigal-González, PradaVásquez, \& Fernández-McCann, 2013) se ha utili- 
zado la diferencia de dos filtros gaussianos DoGs con diferentes desviaciones estándar. Estos filtros se usan para detectar regiones con contraste. En este trabajo se buscan regiones que corresponden a los puntos brillantes de la imagen. La diferencia de filtros gaussianos está dada por (1):

$$
\operatorname{DoG}(x, y)=c_{1} e^{\frac{-\left(x^{2}+y^{2}\right)}{2 \sigma \sigma_{1}^{2}}}-c_{2} e^{\frac{-\left(x^{2}+y^{2}\right)}{2 \sigma 2_{2}^{2}}}
$$

Los resultados demuestran que la relación entre las desviaciones estándar $\sigma_{-}(1)$ y $\sigma_{-}(2)$ es determinante para la detección de los locales máximos. Se considera que para todo I ( $x_{-} \mathrm{i}, \mathrm{y}_{-} \mathrm{i}$ ) $\in \mathrm{V}$, se calcula el promedio $\mu(\mathrm{x}, \mathrm{y})$ y la desviación estándar $\sigma(\mathrm{x}, \mathrm{y})$ y se establece un umbral local $\mathrm{U}(\mathrm{x}, \mathrm{y})$ (Madrigal-González et al., 2013) mediante la ecuación (2):

$$
U(x, y)=B \mu(x, y)+C \sigma(x, y)
$$

Los valores de B y C fueron encontrados en los resultados de la base de datos y sus valores fueron 0.2 y 4.5 , respectivamente. Se analizan distintos valores de $\mathrm{U}(\mathrm{x}, \mathrm{y})$ para cada una de las posiciones $\mathrm{I}\left(\mathrm{x} \_\mathrm{i}, \mathrm{y} \mathrm{i}\right)$ de la región demarcada por la ventana V (Madrigal-Conzález et al., 2013).

\section{FILTRO HOMOMÓRFICO}

Es un filtro poco conocido pero muy útil para manejar el contraste de las mamografías (Cörgel, Sertbas, \& Uçan, 2015). A continuación, se presenta en la ecuación (3):

$$
H(u, v)=\left(y_{H}-y_{L}\right)\left[1-e^{-c\left(\frac{D^{2}(u, v)}{D_{0}^{2}}\right)}\right]+y_{L}
$$

Donde D_0 es una distancia determinada del origen de la transformada y $\mathrm{D}(\mathrm{u}, \mathrm{v})$ es la distancia desde el punto $(\mathrm{u}, \mathrm{v})$ al centro del rectángulo de frecuencias donde la constante c se encarga de controlar la nitidez de la pendiente de la función de filtro cuando transita entre los valores previamente definidos de 0.5 y 2 para los parámetros y_H (alto) y y_L (bajo) respectivamente (Görgel et al., 2015).

FILTRO DE MEDIANA

Es un filtro no lineal utilizado para remover el ruido de una imagen sin distorsionar el resto de pixeles. Además, los filtros de mediana se encargan de eliminar los pixeles aislados que sean brillantes u oscuros. El filtro de mediana adaptativo según (Subash Chandra Bose, Shankar Kumar, \& Karnan, 2012), está diseñado para superar los problemas encontrados con el filtro de mediana estándar. Lo que se diferencia en el filtro adapta- tivo es que el tamaño de la ventana que rodea a cada pixel es variable. Si el valor es un impulso, el tamaño de la ventana se amplia. Si sucede lo contrario, se siguen los siguientes pasos:

1. El centro de pixel de la ventana se evalúa para verificar si es impulso o no.

2. Si da positivo entonces el nuevo valor de ese pixel en la imagen filtrada será la mediana de los pixeles en esa ventana.

3. Si el valor central no es un impulso, entonces el valor del pixel se retiene en la imagen.

4. Si es considerado un impulso, el valor de escala de grises del pixel en la imagen filtrada es el mismo de la imagen de entrada.

El filtro de mediana adaptativo soluciona dos veces más el ruido de impulso de la imagen y reduce su distorsión.

\section{EXTRACCIÓN DE CARACTERÍSTICAS}

Esta etapa define patrones que caracterizan regiones deinterés (ROI), mediantela obtención de métricas que proporcionan una representación y descripción matemática. Para este proceso, se maneja algunos tipos de descriptores tomados de los 36 artículos investigados, resaltando los siguientes algoritmos:

\section{TRANSFORMADA DISCRETA DE COSENO (DCT)}

Este método es utilizado para convertir la señal en sus componentes de frecuencia. La denominada DCT elimina la correlación de la información de la imagen. Las características generadas por la DCT se han utilizado para reconocimiento facial, en donde se extraen los coeficientes relevantes que forman los vectores característicos, reduciendo la dimensión de las características (Talha, 2016). El algoritmo fragmenta la imagen en bloques de 64 bits, es decir; están compuestos de 8 filas y 8 columnas. Este proceso disminuye la pérdida de la calidad de la imagen (Talha, 2016).

Durante la etapa de resultados, se comprobó que las características obtenidas después de la aplicación de DCT, ayudaron a separar las mamografías normales de las sospechosas de cáncer, dando así una sensibilidad alta (Kendall \& Flynn, 2014).

TRANSFORMADA DISCRETA DE ONDÍCULA (DWT)

Este método es una transformación lineal, donde los datos de la imagen se fragmentan 
en sub-imágenes y detallan la información de las sub-bandas verticales $u$ horizontales y diagonales de la mamografía (Talha, 2016). La DWT es un tipo especial de la transformada de Fourier que representa una señal en términos de versiones trasladas y dilatadas de una onda finita (denominada Wavelet). La transformada de Wavelet u ondícula permite variar el tamaño de la ventana de análisis y puede medir las variaciones en tiempo-frecuencia de los componentes espectrales, pero posee una resolución diferente (Mina \& Isa, 2015).

La descomposición de una imagen permite separar valores como "deformaciones intrínsecas" o "factores extrínsecos". Este proceso aisla pequeños cambios que hay entre imágenes. Cuando se aplica este método en 2D, primero se usa el DWT 1D a lo largo de las filas de la imagen, y después los resultados se clasifican en 4 sub-bandas, llamadas bajo-bajo (LL), bajo-alto $(\mathrm{LH})$, alto-bajo $(\mathrm{HL})$ y alto-alto $(\mathrm{HH})$ respectivamente (Subash Chandra Bose et al., 2012).

HISTOGRAMAS

Los histogramas han sido utilizados por John Arévalo y su grupo de investigación (Arevalo, González, Ramos-Pollán, Oliveira, \& Guevara Lopez, 2015). El histograma de gradientes orientados (HOG) y el histograma de la divergencia de gradiente (HCD) en comparación con los descriptores tradicionales generan resultados óptimos tanto en rendimiento como precisión.

HISTOGRAMAS DE ORIENTACIÓN GRADIENTE (HOG) Este histograma es utilizado especialmente para la detección y reconocimiento de caracteres numéricos. Su funcionamiento es calcular los gradientes de la imagen, luego divide la imagen en celdas cuadradas, es decir; en una cuadrícula de bloques, donde cada bloque está descrito por un histograma de orientación de gradiente para cada uno de los vectores. La mayor magnitud de los gradientes tendrá un impacto considerado sobre el histograma. Dentro del proceso donde las celdas son agrupadas en un bloque, los histogramas que están en el bloque son normalizados y después concatenados formando así un descriptor de HOG (Arevalo et al., 2015).

HISTOGRAMA DE LA DIVERGENCIA DE GRADIENTE (HGD) Este histograma describe imágenes mediante la distribución de la divergencia de gradiente donde las imágenes son divididas en regiones concéntricas y cada región se describe por un histograma. La forma de representarlas es con dos variables i, j, y el ángulo se mide entre el vector de gradiente de la intensidad en $\mathrm{i}, \mathrm{j}$ y un vector que se dirige hacia el núcleo de la imagen con origen en i, j (Arevalo et al., 2015).

FILTROS GABOR

Este filtro es el más utilizado en el ámbito de preprocesamiento de la imagen debido a la eficiencia de la descripción de textura de las imágenes de mamografías según (Barrena \& Valls, 2014). El propósito general del filtro es la descomposición de una imagen en múltiples escalas y orientaciones para lograr el análisis de los patrones de texturas (Khan et al. 2016; Tusa et al. 2014). Según (Khan et al., 2016), los filtros Gabor representan diferentes escalas y orientaciones en micropatrones de ROIs. La fórmula general de los filtros de Gabor 2D está compuesta por un centro gaussiano modulado por una exponencial compleja (Barrena \& Valls, 2014; Torrents-Barrena, Puig, Melendez, \& Valls, 2016).

TRANSFORMACIÓN ESFÉRICA DE ONDÍCULA (SWT) Este método es considerado como un aporte a la investigación, generando combinaciones multiescalares y analizando la deficiencia en algunos procesos convencionales de ondícula (Görgel et al., 2015). Se ha comprobado que la transformación esférica de ondícula proporciona una detección mucha más eficiente y son funciones que representan una señal en múltiples niveles de detalle, llamadas resoluciones (Görgel, Sertbas, \& Ucan, 2013)

MATRIZ DE CO-OCURRENCIA DE NIVEL GRIS (GLCM)

Es una técnica estadística muy importante para la obtención de información de texturas de imágenes de segundo orden. Son medidas que considera la relación de co-ocurrencia entre grupos de dos pixeles de la imagen original a una distancia dada. Además, se encarga de la distribución espacial de los niveles de gris en una imagen. Su función establece que i es el número de filas y j es el número de columnas, Vi,j es el valor de la celda (i,j) en la ventana; $\mathrm{Pi}, \mathrm{j}$ es la probabilidad en la celda (i,j); $N$ es el número de filas y columnas. Las medidas de texturas se derivan de la matriz normalizada y se denominan: homogeneidad, contraste, disimilaridad, GLCM media, desviación estándar, entropía, correlación, segundo momento angular (ASM, por sus siglas en Inglés) (Malar et al., 2012).

$$
P_{i, j}=\frac{v_{i, j}}{\sum_{i, j=0}^{N-1} v_{i, j}}
$$


La matriz co-ocurrencia según (Padmanabhan \& Sundararajan, 2012), se ha utilizado para calcular las características de texturas que describen la probabilidad conjunta de que los niveles de grises, se producen en una dirección 0 a una distancia d (en número de pixeles). La probabilidad conjunta describe la frecuencia con la que se produce un valor de pares de nivel de gris entre pares de pixeles con una relación espacial relativa. En (Pérez, Benalcázar, Tusa, Rivas, \& Conci, 2017), se utiliza este tipo de características de textura y una red neuronal artificial para clasificar tres categorías de tejidos: normal, benigno y maligno; con una precisión de $84.72 \%$, utilizando el lenguaje de programación MATLAB (San Martin Cuenca \& Tusa, 2015).

MULTIRESOLUCIÓN DE PATRONES LOCALES BINARIOS (LBP)

Este método de multiresolución consiste en la comparación del pixel central con los vecinos, donde el pixel central es tomado como el umbral con respecto a sus vecinos. Al comparar el pixel central con el vecino se le asigna un valor de uno, si el vecino es mayor o igual, caso contrario se le asigna un valor de cero. El operador LBP produce $2 \mathrm{n}$ valores diferentes, donde $\mathrm{n}$ es el número de pixeles en el vecindario del pixel central. Luego se realiza una sumatoria de los diferentes pesos obteniendo la representación de LBP de pixel (Choi, Kim, Choi, \& Ro, 2012).

Este operador permite modificar los tamaños del área de interés y las vecindades para el pixel de interés. La derivación consiste en el establecimiento de los parámetros $\mathrm{R}$ y $\mathrm{P}$, donde $\mathrm{R}$ corresponde a la distancia o radio tomado a partir del pixel origen y $\mathrm{P}$ es el número de vecinos utilizados para el cálculo de LBP. La distancia y la distribución de los vecinos se construyen a partir de una circunferencia simétrica. Para valores de $\mathrm{R}>0$ y $\mathrm{P}>1$, se pueden obtener diferentes medidas de textura del área de interés, los cuales se denominan patrones uniformes debido a que contienen como máximo dos transiciones bit a bit de 0 a 1 o viceversa. La razón latente de esto es que el nivel de gris cambia los pesos que suelen ser constantes y no de forma brusca (Choi et al., 2012).

ANÁLISIS DE COMPONENTES PRINCIPALES (PCA)

Es un método conocido por los estudios realizados y enfocados a una reducción masiva de falsos positivos (FPR, por sus siglas en inglés). Los patrones LBPs elevan la eficiencia en la reducción de falsos positivos en diferentes regiones de interés. Si se comparan los resultados con LBPS, este presenta mejores promedios frente al PCA en dos dimensiones (2DPCA) (Torrents-Barrena et al. 2016)

PCA una transformación lineal bajo la teoría de mapas de alta dimensionalidad. El PCA es más eficiente si es utilizado en clasificación para facilitar la detección por la exactitud y el bajo tiempo computacional. Según (TorrentsBarrena et al. 2016), su estructura se basa en dimensiones, donde la primera dimensión es la componente principal que posee la mayor varianza, mientras que las demás componentes mantienen valores de varianza menores. La reducción dimensional brinda buenos resultados con el riesgo de disminuir la precisión.

\section{LA TRANSFORMADA DE HOUGH}

Es utilizada en la detección de curvas y ruido en una imagen. Primero, los pixeles de los bordes en la imagen se transforman a una imagen binaria. Su objetivo es detectar puntos alineados que satisfacen la ecuación de la recta. En el experimento de (Xie, Li, \& Ma, 2016), se aplica la transformada Hough lineal para la detección de músculo pectoral, y el límite de la masa se lo detecta con Hough circular.

\section{FILTRO ESPEJO EN CUADRATURA (OMF)}

La imagen se desfragmenta mediante un banco de filtros en sub-bandas frecuenciales que se codifican por separado. Gran parte de la información está comprendida en bandas frecuenciales bajas y necesitan mayor resolución, mientras que las frecuencias altas requieren una menor resolución. Al sumarse las ganancias obtenidas de los dos filtros, da un valor constante para evitar una banda frecuencial atenuada. Este proceso se denomina filtro espejo (QMF), dividiendo la señal en bandas para sub-muestrearlas (Pérez et al., 2016).

\section{FILTRO DE ONDÍCULA}

Este filtro funciona a varias escalas mediante la aplicación de diferentes frecuencias. La ondícula ortogonal descompone la imagen en un conjunto de coeficientes independientes que evitan la redundancia en la información a través de características eficientes. La mamografía se descompone en cuatro niveles y las características extraídas para la clasificación son la energía y la norma infinita para las cuatro componentes (Malar et al., 2012).

Es un método eficiente para la extracción de características de multiresolución asociadas 
a componentes de mayor frecuencia como las microcalcificaciones (Zhang, Chen, Zhang, \& Zhang, 2012).

\section{MÉTRICAS DE LAS LEYES DE ENERGÍA DE TEXTURA (LAWS)}

Este método nos ayuda a extraer características secundarias de la imagen mediante la utilización de filtros de máscara. LAWS se elige por su capacidad superior para extraer la textura de una imagen. Entre las características tenemos el color, forma o tamaño. La textura es muy importante para la clasificación y para la obtención de información de la imagen. LAWS utiliza un conjunto de máscaras de convolución de $5 \times 5$ para calcular la energía de textura, que se representa por un vector de nueve números para cada pixel analizado de la imagen. Existen 4 características principales que se pueden analizar: borde, nivel, mancha y ondulación (Setiawan, Elysia, Wesley, \& Purnama, 2015).

Las características son representadas con una letra mayúscula delante del nombre del vector. Por ejemplo, el cálculo del vector L5 es resultado del promedio local ponderado, E5 vector indica bordes, el S5 detecta la mancha y $\mathrm{R} 5$ detecta el vector onda. LAWS resta el promedio local de cada pixel para producir una nueva imagen procesada, en la que la intensidad media de cada vecindario está próxima a cero (Setiawan et al., 2015).

El resultado muestra que LAWS proporciona una mejor precisión que otros métodos similares, como el GLCM. LAWS proporcionan $93.90 \%$ precisión para normal-anormal y $83.30 \%$ para clasificación benigno-maligna, mientras que GLCM solo proporciona $72.20 \%$ de precisión para normal-anormal y 53.06\% para clasificación benigno-maligno (Setiawan et al., 2015).

\section{ALGORITMOS DE CLASIFICACIÓN MÁQUINA DE APRENDIZAJE EXTREMA ELM}

El algoritmo ELM está incluido en SLFN (siglas del inglés: Single Layer Feed Forward), cuya velocidad de aprendizaje es mil veces más rápida que las redes convencionales de alimentación anticipada. Los pesos de entrada y los sesgos de capa oculta se pueden asignar al azar si las funciones de activación en la capa oculta son infinitamente diferenciables pues se dice que tienen mejor generalización (Xie, Li, \& Ma, 2016).

Con $\mathrm{N}$ neuronas ocultas y una función de activación $\mathrm{g}(\mathrm{x})$, el ELM puede resumirse como la asignación de los pesos de entrada y sesgos aleatoriamente, de acuerdo con la función de densidad de probabilidad. En primer lugar, se calcula la matriz de salida de la capa oculta $\mathrm{H}$, luego se calcula los pesos $\beta=\mathrm{H}^{\prime} \mathrm{T}$ donde $\mathrm{H}^{\prime}$ es la generalización inversa Moore-Penrose de la matriz $\mathrm{H}$.

Para el funcionamiento de ELM se necesita definir los parámetros a utilizar como el número de neuronas ocultas y la función de activación. Se han llevado a cabo estudios de investigación para optimizar los parámetros anteriores por medio de la variación del número de neuronas ocultas de 5 a 80 ingresos como número máximo para diferentes funciones de activación.

Se ha probado que ELM posee mejor rendimiento frente a otros clasificadores existentes como Bayes, Bayes ingenuo y las máquinas de vectores de soporte para tres diferentes características extraídas: Matriz de dependencia espacial de nivel gris (CLSDM, por sus siglas en Inglés), filtro Gabor y técnicas de ondícula.

\section{MÁaUINAS DE VECTORES DE SOPORTE (SVM)}

Es un clasificador de gran alcance que es capaz de representar relaciones entre las características con los modelos, generalizando una amplia gama de aplicaciones en el mundo real, incluyendo el reconocimiento y detección de rostros e identificación de voces (Zhang et al., 2012). Muchas de las características suelen ser irrelevantes o redundantes, aumentando la complejidad del espacio de búsqueda. Las SVM son muy sensibles al ruido presente en los datos y son propensos a la generalización excesiva. Además, se puede degradar el rendimiento de la clasificación con el fin de atender este déficit (Khan et al., 2016). El clasificador toma la entrada del vector que es mapeado en un espacio de características de forma lineal o no lineal (Barrena, 2014), que está relacionado con la función del núcleo. Luego, las características se dividen en dos clases (Kamalakannan, Thirumal, Vaidhyanathan, \& Mukeshbhai, 2015) por el hiperplano, donde van alojados los vectores de soporte (Zakeri, Behnam, \& Ahmadinejad, 2012).

Las SVM comprenden un método de estimación en el proceso de clasificación de datos. Asi, se forma un hiperplano como la superficie de decisión de tal manera que el margen de separación es entre el negativo y el positivo. Las SVM utilizan las funciones del núcleo para predecir los valores resultantes (Görgel et al., 2015).

Otro experimento del clasificador SVM donde utiliza una base radial como núcleo, se construyó para evaluar la eficiencia de las características de la propuesta de Multiresolución LBP para la clasificación de masas. Para lograr 
el menor error de generalización (TorrentsBarrena et al., 2015), se ha aplicado 10 veces la validación cruzada a los valores de las características en el conjunto de formación con el propósito de optimizar tanto el parámetro asociado de la función del núcleo, como el parámetro de regularización. Las puntuaciones de salida SVM se utilizaron como variable de decisión en el receptor de características operativas y asi evaluar el rendimiento de la clasificación (Choi et al., 2012).

K VECINOS MÁS CERCANOS (KNN) Según el estudio de (Madrigal-González et al., 2013), el clasificador $K$ vecinos más cercanos (KNN) es usado para determinar el grado de malignidad o benignidad de las microcalcificaciones. En la etapa de clasificación se le asigna la clase de normal o anormal a cada una de las microcalcificaciones sospechosas obtenidas durante el proceso de segmentación. Además, emplea funciones de distancias como parámetros de asignación de la clase. Durante la fase de extracción de características, fueron creadas un total de 7 métricas para cada una de las microcalcificaciones sospechosas. Posteriormente, se procedió a calcular la distancia de Mahalanobis. En este estudio, se analizó la sensibilidad del algoritmo KNN, aumentando el valor umbral $\mathrm{k}$ a ser menor o igual que $\mathrm{K}$ con el fin de reducir las muestras de entrenamiento a una pequeña fracción de la original.

RED NEURONAL ARTIFICIAL (ANN)

Es un paradigma de procesamiento de la información que se compone de un gran número de elementos de procesamiento altamente interconectados (neuronas) que trabajan al unísono para resolver problemas específicos. Una ANN está configurada para la aplicación específica de reconocimiento o clasificación de datos a través de un proceso supervisado (Subash Chandra Bose et al., 2012).

Esta red de propagación de alimentación posee dos capas con funciones de transferencia sigmoide tanto en la capa oculta como en la capa de salida, 18 nodos de entrada y se entrena con diferentes cantidades de neuronas en la capa oculta en cada proceso de formación, alcanzando el mejor resultado con 10 nodos ocultos. Existen dos pasos de clasificación: el primero considera los datos normales y anormales y el segundo, los datos anormales recogidos de la primera clasificación. Así, se repite la clasificación para verificar si son benignos o malignos
(Setiawan et al., 2015). ANN entrena a las neuronas artificiales usando una entrada definida. Se calcula el mejor peso de cada neurona, de modo que el error mínimo pueda ser alcanzado. La tasa de error puede ser estimada usando el error cuadrático medio (MSE, por sus siglas en Inglés) (Pratiwi, Alexander, Harefa, \& Nanda, 2015).

Existen mejoras de este clasificador, como la red neuronal de propagación hacia atrás (BPNN, por sus siglas en Inglés). Este clasificador ajusta el peso de cada neurona durante el proceso de entrenamiento con el fin de minimizar el MSE. Algunos estudios han demostrado que esta red es más robusta (Pérez et al., 2017; Pratiwi et al., 2015).

\section{BAYESIANO INGENUO}

Este clasificador fue utilizado para calcular la probabilidad de que la imagen mamográfica principal contenga una anormalidad. El esquema de clasificación debe dar una medida de confianza de que la imagen contiene una anomalía. Los clasificadores bayesianos ingenuos sencillos no generan estas medidas de confianza. Por ello, se construyó una red de clasificadores bayesianos ingenuos que se adaptara a las necesidades. El rendimiento de la red en la clasificación de imágenes conocidas, se utilizó para calcular una estadística de confianza de clasificación (Kendall, Barnett, \& Chytyk-Praznik, 2013).

Para la evaluación del rendimiento de la red, se utilizó validación cruzada dejando uno afuera. Esta técnica separa los datos de forma que para cada iteración tengamos una sola muestra para los datos de prueba y todo el resto conforma los datos de entrenamiento. Las características calculadas a partir de mapas de ondícula de una imagen, formaron las entradas para el clasificador. Luego se construyó la red de clasificadores pasando las salidas de las imágenes normales y sospechosas de un clasificador a otros clasificadores adicionales para su posterior análisis (Kendall et al., 2013). Los autores en (Padmanabhan \& Sundararajan, 2012) calculan características estadísticas y de textura, con el objetivo de analizar el efecto en la etapa de entrenamiento de forma separada. El propósito es la identificación de tres tipos de clases: normal, benigno y maligno.

\section{RED NEURONAL CONVOLUCIONAL (CNN)}

Las redes neuronales convolucionales son un tipo de red neuronal que se caracteriza por la compartición de los pesos que se convolucionan a través de la entrada mediante una ventana de 
movimiento (Wahab, Khan, \& Lee, 2017). Esta propiedad convolucional aplicada en una capa de agrupación alcanza invariancia traslacional, que se adapta especialmente a las imágenes. Es importante destacar que la red convolucional trabaja con cuatro capas que se detallan a continuación: capa convolucional, la función de activación, capa de agrupación, capa de salida (Lévy \& Jain, 2016).

La capa convolucional utiliza un filtro o una matriz de pesos, que se desplaza a través de las entradas de la capa anterior. De este modo, las salidas resultan de calcular el producto punto entre los pesos y las entradas (Qayyum, Anwar, Awais, \& Majid, 2017). Luego se aplica la función de activación que introduce efectos no lineales sobre los elementos con el fin de producir un mapa de características, donde cada una de las entradas se considera como la salida de una sola neurona asociada a una pequeña región local de entrada (Wahab et al., 2017).

La función de activación es una función no lineal que se aplica a los elementos de convolución (Arevalo et al., 2015). La capa de agrupación reduce la complejidad computacional de una CNN mediante la aplicación de una o más capas de agrupación a los mapas de características producidos por las capas convolucionales, disminuyendo el tamaño de los mapas. La capa de salida posee un número de nodos igual al número de clases en el conjunto de datos (Wahab et al., 2017). Los autores en (Soriano et al., 2018) utilizan una CNN para clasificar un banco de 1070 mamogramas obtenidos de la base de datos DDSM entre las clases benigno y maligno. El resultado de esta experiencia genera una precisión del $85 \%$. Una segunda experiencia propuesta por (Moya et al., 2017), utiliza 1000 mamogramas divididos en 5 clases, registrando una precisión del 97\%.

\section{RED NEURONAL DE ONDÍCULA (WNN)}

La red neuronal de ondícula es un tipo especial de red neuronal con funciones de activación. Tienen generalmente una estructura de retroalimentación hacia adelante con una capa oculta. Las funciones de activación se extraen de una familia de ondículas ortonormales. Las funciones de activación de ondículas más comunes son las funciones Gaussiana, Gabor y ondículas Haar (Khan et al., 2017).

RED NEURONAL DE FUNCIÓN DE BASE RADIAL (RBFNN) Esta red neuronal posee una capa de neuronas de función de base radial y una capa de salida con un nodo para cada categoría o clase de datos. Los autores en (Pratiwi et al., 2015) entrenan esta red con 4 tipos de características: ASM, correlación, sumatoria de la entropía y sumatoria de la varianza. La capa oculta tiene un número variable de neuronas, de las cuales se han utilizado 84 imágenes, considerando que el número de instancias en el conjunto de entrenamiento es 85 (Pratiwi et al., 2015). La capa de salida consiste en un conjunto de nodos que se clasifican de acuerdo a dos reglas:

$$
\begin{aligned}
& \text { si } x>k \text { entonces } x=k \\
& \text { si } x<j \text { entonces } x=j
\end{aligned}
$$

Los valores de $\mathrm{k}$ y $\mathrm{j}$ representan valores umbrales superior e inferior, respectivamente. La distancia euclidiana se calcula a partir del punto que se evalúa al centro de cada neurona y se aplica una función de base radial (RBF) a la distancia para calcular el peso de cada neurona. Ceneralmente, la función Gaussiana es seleccionada como función de base radial o función núcleo en la mayoría de los estudios (Pratiwi et al., 2015).

\section{ALGORITMOS HÍBRIDOS DE CLASIFICACIÓN}

TABLA 3. DETALLES DE LOS ALGORITMOS HÍBRIDOS DE
CLASIFICACIÓN




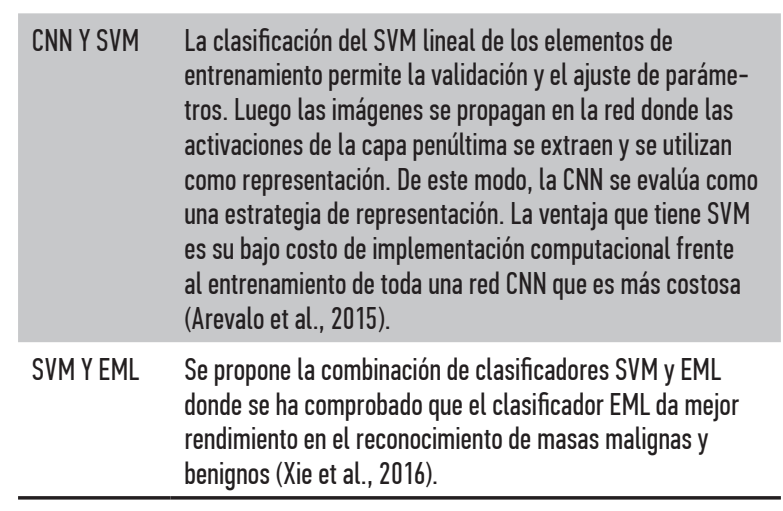

MÉTRICAS

Las métricas son utilizadas para evaluar si la clasificación de un sistema de decisión es $\mathrm{CO}^{-}$ rrecta o incorrecta con el propósito de definir el mejor rendimiento y hacer una predicción más realista. Las métricas más utilizadas dentro de la detección de anomalías para evaluar el desempeño de los clasificadores son: el receptor de características operativas (ROC), que comprende Sensibilidad y Especificidad; y la precisión.

CURVA ROC

El área bajo la curva denominada antiguamente como AUC, debido a su gran acogida y su eficiencia es considerada como el método estándar para evaluar el rendimiento de los sistemas de decisión predictivos. La curva ROC se representa con la sensibilidad que indica la función de la tasa verdadera positiva y la especificidad la función de la tasa de positivos falsos (Xie et al., 2016). Es importante tener en consideración los siguientes casos:

- TP es el caso positivo verdadero (Khehra \& Pharwaha, 2016).

- TN son los casos negativos verdaderos (Kamalakannan et al., 2015).

- FN instancia clasificada como negativo falso (Torrents-Barrena et al., 2015).

- FP instancia clasificada como positivo falso (Torrents-Barrena et al., 2015).

PRECISIÓN

La precisión aplicada a la detección de anomalías indica la confiabilidad que debemos tener en los resultados (Fonseca et al., 2015). Es decir, es la manera de evaluar el número de casos positivos o negativos que existen dentro del conjunto de imágenes para adquirir un nivel de precisión (Malar et al., 2012).

CONCLUSIONES

La revisión exhaustiva de los esquemas de clasificación ha considerado un conjunto de 36 artí- culos científicos que han sido los elementos para identificar las bases de datos más recurrentes por los investigadores. En su gran mayoría, las bases de mamografías MIAS y DDSM presentan un porcentaje de $90 \%$ de presencia entre los artículos estudiados. Los métodos de filtrado para la etapa de preprocesamiento señala a la ecualización del histograma (escala de grises) con cinco artículos de coincidencias. La etapa de algoritmos de extracción de características recoge los métodos convencionales en cinco artículos como la transformada de coseno discreto y la transformada de ondícula discreta. El filtro de Gabor registra tres coincidencias de autores, entre otros filtros mencionados anteriormente que han demostrado rendimientos aceptables. La etapa de algoritmos de clasificación, según el estudio, el clasificador más utilizado son las máquinas de vectores de soporte (SVM). Además, se han encontrado algunas combinaciones de clasificadores convencionales que han marcado una notoria precisión en los resultados. También se ha observado la presencia de los clasificadores convencionales (ANN, RNA, KNN).

Algunos autores han dedicado su atención para mejorar esos clasificadores convencionales, y adaptarlos a sus necesidades como es el caso de RBFNN, WNN. Se ha observado que las redes CNN han tenido una gran acogida en los últimos cinco años debido a su buen rendimiento. La presencia de este clasificador ha mostrado eficiencia en los resultados y su proceso consta en extraer las características de la imagen siempre y cuando existe una base de datos con abundante información. En base a las métricas de evaluación podemos conocer si el resultado de la predicción es exacto, validando el desempeño de los algoritmos. Existe una métrica conocida anteriormente como AUC que en la actualidad se conoce como la curva de ROC (el receptor de características operativas) y la precisión que intenta cuantificar el rendimiento de los algoritmos.

\section{AGRADECIMIENTO}

Los autores agradecen a la Dra. María Pérez, docente de la Escuela Politécnica Nacional, por su enorme gestión para la realización de este trabajo en el marco del proyecto REDU PREREDU-2016-013 "Desarrollo de un sistema inteligente para análisis automático de lesiones mamarias utilizando técnicas de inteligencia artificial y otras técnicas computacionales de análisis de imágenes digitales” 


\section{REFERENCIAS BIBLIOGRÁFICAS}

Arevalo, J., González, F. A., Ramos-Pollán, R., Oliveira, J. L., \& Guevara Lopez, M. A. (2015). Representation learning for mammography mass lesion classification with convolutional neural networks. Computer Methods and Programs in Biomedicine, 127, 248-257. https://doi. org/10.1016/j.cmpb.2015.12.014

Barrena, J. T., \& Valls, D. P. (2014). Tumor Mass Detection through Gabor Filters and Supervised PixelBased Classification in Breast Cancer. University Rovira, Virgil.

Choi, J. Y., Kim, D. H., Choi, S. H., \& Ro, Y. M. (2012). Multiresolution Local Binary Pattern texture analysis for false positive reduction in computerized detection of breast masses on mammograms, 8315, 83152B. https://doi.org/10.1117/12.911137

Díaz Sotolongo, A., Cárcel Carrasco, F. J., Martín Alfonso, J. A., \& Pérez Pérez, N. (2014). Combinación de técnicas de procesamiento digital de imágenes y geometría analítica para la detección de lesiones patológicas en imágenes de mamografía. 3C Tecnología, 3(3), 180-191.

Eltoukhy, M. M., \& Faye, I. (2013). An adaptive threshold method for mass detection in mammographic images. IEEE ICSIPA 2013 - IEEE International Conference on Signal and Image Processing Applications, 374-378. https://doi.org/10.1109/ ICSIPA.2013.6708036

Fonseca, P., Mendoza, J., Wainer, J., Ferrer, J., Pinto, J., Guerrero, J., \& Castaneda, B. (2015). Automatic breast density classification using a convolutional neural network architecture search procedure. SPIE Medical Imaging, 9414, 94-108. https://doi. org/10.1117/12.2081576

Görgel, P., Sertbas, A., \& Ucan, O. N. (2013). Mammographical mass detection and classification using Local Seed Region Growing-Spherical Wavelet Transform (LSRG-SWT) hybrid scheme. Computers in Biology and Medicine, 43(6), 765-774. https:// doi.org/10.1016/j.compbiomed.2013.03.008

Görgel, P., Sertbas, A., \& Uçan, O. N. (2015). Computer-aided classification of breast masses in mammogram images based on spherical wavelet transform and support vector machines. Expert Systems, 32(1), 155-164. https://doi.org/10.1111/ exsy. 12073

Jankulovski, B., Kitanovski, I., Trojacanec, K., Dimitrovski, I., \& Loskovska, S. (2012). Mammography image classification using texture features, (Ciit), 3-6.

Kamalakannan, J., Thirumal, T., Vaidhyanathan, A., \& Mukeshbhai, K. D. (2015). Study on different classification technique for mammogram image. IEEE International Conference on Circuit, Power and Computing Technologies, ICCPCT 2015, (c). https://doi.org/10.1109/ICCPCT.2015.7159456

Kendall, E. J., Barnett, M. G., \& Chytyk-Praznik, K. (2013). Automatic detection of anomalies in screening mammograms. BMC Medical Imaging, 13(1), 43. https://doi.org/10.1186/1471-2342-13-43

Kendall, E. J., \& Flynn, M. T. (2014). Automated breast image classification using features from its discrete cosine transform. PLoS ONE, 9(3), 8-15. https://doi.org/10.1371/journal.pone.0091015

Khan, M. M., Mendes, A., Zhang, P., \& Chalup, S. K. (2017). Evolving multi-dimensional wavelet neural networks for classification using Cartesian Genetic Programming. Neurocomputing, 247, 3958. https://doi.org/10.1016/j.neucom.2017.03.048

Khan, S., Hussain, M., Aboalsamh, H., Mathkour, H., Bebis, G., \& Zakariah, M. (2016). Optimized Gabor features for mass classification in mammography. Applied Soft Computing Journal, 44, 267280. https://doi.org/10.1016/j.asoc.2016.04.012

Khehra, B. S., \& Pharwaha, A. P. S. (2016). Classification of clustered microcalcifications using MLFFBP-ANN and SVM. Egyptian Informatics Journal, 17(1), 11-20. https://doi.org/10.1016/j. eij.2015.08.001

Kowal, M., Filipczuk, P., Obuchowicz, A., Korbicz, J., \& Monczak, R. (2013). Computer-aided diagnosis of breast cancer based on fine needle biopsy microscopic images. Computers in Biology and Medicine, 43(10), 1563-1572. https://doi.org/10.1016/j. compbiomed.2013.08.003

Lévy, D., \& Jain, A. (2016). Breast Mass Classification from Mammograms using Deep Convolutional Neural Networks. Retrieved from http://arxiv.org/ abs/1612.00542

Madrigal-González, C. A., Prada-Vásquez, R., \& Fernández-McCann, D. S. (2013). Detección Automática de Microcalcificaciones en una Mamografía Digital, Usando Técnicas de Inteligencia Artificial. Tecno Lógicas, 0(0), 743-756.

Malar, E., Kandaswamy, A., Chakravarthy, D., \& Giri Dharan, A. (2012). A novel approach for detection and classification of mammographic microcalcifications using wavelet analysis and extreme learning machine. Computers in Biology and $\mathrm{Me}^{-}$ dicine, 42(9), 898-905. https://doi.org/10.1016/j. compbiomed.2012.07.001

Mina, L. M., \& Isa, N. A. M. (2015). Microcalcification diagnosis in digital mammograms based on wavelet analysis and neural networks. 2015 IEEE International Conference on Control System, Computing and Engineering (ICCSCE), 7-12.

Moya, E., Campoverde, E., Tusa, E., RamirezMorales, I., Rivas, W., \& Mazon, B. (2017). Multi-category Classification of Mammograms by 
Using Convolutional Neural Networks. 2017 International Conference on Information Systems and Computer Science (INCISCOS), 133-140. https:// doi.org/10.1109/INCISCOS.2017.56

Nariño, U. A. (2016). Técnicas de umbralización para el procesamiento digital de imágenes de GEMFoils Thresholding techniques for digital image processing of GEM-Foils, 21(4), 352-359.

Padmanabhan, S., \& Sundararajan, R. (2012). Texture and Statistical Analysis of Mammograms : A Novel Method to Detect Tumor in Breast Cells, 157-160.

Pérez, M., Benalcázar, M. E., Tusa, E., Rivas, W., \& Conci, A. (2017). Mammogram classification using back-propagation neural networks and texture feature descriptors. 2017 IEEE Second Ecuador Technical Chapters Meeting (ETCM), 1-6.

Pérez, M. G., Benalcazar, M., Conci, A., \& Fiallos, C. (2016). Detección de patologías de la mama mediante filtros multirresolución, 187-193.

Pérez, N., Valdés, J. A., Guevara, M. A., Rodríguez, L. A., \& Molina, J. M. (2007). Set of methods for spontaneous ICH segmentation and tracking from CT head images. Iberoamerican Congress on Pattern Recognition (CIARP), 212-220.

Pratiwi, M., Alexander, Harefa, J., \& Nanda, S. (2015). Mammograms Classification Using Gray-level Co-occurrence Matrix and Radial Basis Function Neural Network. Procedia Computer Science, 59(Iccsci), 83-91. https://doi.org/10.1016/j. procs.2015.07.340

Qayyum, A., Anwar, S. M., Awais, M., \& Majid, M. (2017). Medical Image Retrieval using Deep Convolutional Neural Network. Neurocomputing, 0, 1-13. https://doi.org/10.1016/j.neucom.2017.05.025

San Martin Cuenca, H. , \& Tusa, E. (2015). Fundamentos de programación para ciencias e ingeniería. Machala: Universidad Técnica de Machala.

Setiawan, A. S., Elysia, Wesley, J., \& Purnama, Y. (2015). Mammogram Classification using Law's Texture Energy Measure and Neural Networks. Procedia Computer Science, 59(Iccsci), 92-97. https://doi.org/10.1016/j.procs.2015.07.341

Soriano, D., Aguilar, C., Ramirez-Morales, I., Tusa, E., Rivas, W., \& Pinta, M. (2018). Mammogram Classification Schemes by Using Convolutional Neural Networks. In M. Botto-Tobar, N. EsparzaCruz, J. León-Acurio, N. Crespo-Torres, \& M. Beltrán-Mora (Eds.), Technology Trends (pp. 71-85). Cham: Springer International Publishing.

Subash Chandra Bose, J., Shankar Kumar, K. R., \& Karnan, M. (2012). Detection of Microcalcification in Mammograms using Soft Computing Techniques. European Journal of Scientific
Research, 86(1), 103-122. Retrieved from http:// www.europeanjournalofscientificresearch.com

Talha, M. (2016). Classification of mammograms for breast cancer detection using fusion of discrete cosine transform and discrete wavelet transform features ., 27(2), 322-327.

Thomaz, R. L., Carneiro, P. C., \& Patrocinio, A. C. (2017). Feature extraction using convolutional neural network for classifying breast density in mammographic images. SPIE $\mathrm{Me}^{-}$ dical Imaging, 10134, 101342M. https://doi. org/10.1117/12.2254633

Torre, L. A., Bray, F., Siegel, R. L., Ferlay, J., Lortettieulent, J., \& Jemal, A. (2015). Global Cancer Statistics, 2012. CA: A Cancer Journal of Clinicians., 65(2), 87-108. https://doi.org/10.3322/caac.21262.

Torrents-Barrena, J., Puig, D., Melendez, J., \& Valls, A. (2016). Computer-aided diagnosis of breast cancer via Gabor wavelet bank and binary-class SVM in mammographic images. Journal of Experimental \& Theoretical Artificial Intelligence: JETAI, 28(1-2), 295-311.

Tusa, E., Reynolds, A., Lane, D. M., Robertson, N. M., Villegas, H., \& Bosnjak, A. (2014). Implementation of a fast coral detector using a supervised machine learning and Gabor Wavelet feature descriptors. IEEE Sensor Systems for a Changing Ocean (SSCO)., 1-6. https://doi.org/10.1109/ SSCO.2014.7000371

Wahab, N., Khan, A., \& Lee, Y. S. (2017). Two-phase deep convolutional neural network for reducing class skewness in histopathological images based breast cancer detection. Computers in Biology and Medicine, 85, 86-97. https://doi.org/10.1016/j. compbiomed.2017.04.012

Xie, W., Li, Y., \& Ma, Y. (2016). Breast mass classification in digital mammography based on extreme learning machine. Neurocomputing, 173, 930-941. https://doi.org/10.1016/j.neucom.2015.08.048

Zakeri, F. S., Behnam, H., \& Ahmadinejad, N. (2012). Classification of benign and malignant breast masses based on shape and texture features in sonography images. Journal of Medical Systems, 36(3), 1621-1627. https://doi.org/10.1007/s10916010-9624-7

Zhang, L., Chen, Y., Zhang, F., \& Zhang, L. (2012). Detection of clustered pleomorphic micro-calcifications in digital mammograms. Proceedings - 2012 International Conference on Biomedical Engineering and Biotechnology, ICBEB 2012, (3), 768-771. https://doi.org/10.1109/iCBEB.2012.130 